\title{
Enhanced critical current density in MgB2 prepared by reaction of MgB4 and Mg
}

\begin{abstract}
Instead of reacting $\mathrm{B}$ and $\mathrm{Mg}$ powders, $\mathrm{MgB} 4$ was used as precursor to react with $\mathrm{Mg}$ to obtain the polycrystalline samples of nominal composition $\operatorname{MgxB} 2(\mathrm{x}=1.2,1.5$, and 1.7). Upon increasing the sintering temperature to $750^{\circ} \mathrm{C}$, the reaction between $\mathrm{MgB} 4$ and $\mathrm{Mg}$ was thermodynamically intensified leading to the formation of larger $\mathrm{MgB} 2$ weight fraction as estimated from X-ray diffraction (XRD) data using the Rietveld method. Despite no further improvement to the weight fraction of this phase by increasing the excess nominal $\mathrm{Mg}$, it was shown that the critical current density (Jc) was enhanced by almost one order of magnitude. At $5 \mathrm{~K}, 2 \mathrm{~T}$, Jc for the sample $\mathrm{Mg} 1.5 \mathrm{~B} 2$ is estimated to be $4.5 \times 105 \mathrm{Acm}-2$. The enhancement of $\mathrm{Jc}$ is attributed to the collective effect of improved grain connectivity and flux pinning by unreacted $\mathrm{Mg}$. Incorporation of nano-SiC for reaction resulted in a more gradual drop of Jc with applied magnetic field. Hence, Jc can be tailored to meet the range of fields intended for various applications via optimization of excess nominal $\mathrm{Mg}$ and dopant additions.
\end{abstract}

Keyword: Critical current density; MgB2; MgB4; SiC; Superconductivity. 